\title{
Self-paced approach in synergistic model for supporting and testing students
}

\author{
The transition from Secondary School to University
}

\author{
Alice Barana \\ alice.barana@unito.it \\ Alessandro Bogino \\ alessandro.bogino@unito.it \\ Michele Fioravera \\ michele.fioravera@unito.it \\ Francesco Floris \\ francesco.floris@unito.it
}

\author{
Marina Marchisio \\ marina.marchisio@unito.it \\ Lorenza Operti \\ lorenza.operti@unito.it \\ Sergio Rabellino \\ sergio.rabellino@unito.it \\ University of Turin \\ Turin, Italy
}

\begin{abstract}
This paper shows the model developed by the University of Turin to support students that must face the transition from the last year of secondary school to the first year of University. Integrations that are specifically designed for Learning Management Systems help sustain three effective actions conducted in synergy: increase students' awareness in the choice of the future course of study, support them in taking the admission tests and the first-year exams, allow the autonomous administration of admission tests led by the University. The methodological strategies adopted are presented and discussed based on the analysis of the data of the years 2015 and 2016.
\end{abstract}

Keywords-automatic assessment; learning management system; self-paced MOOC; test for the assessment of minimum requirements; university guidance

\section{INTRODUCTION}

Italian universities develop study programs in compliance with provisions of current laws [1], according to which, teaching regulations define requirements for accessing courses and verification procedures. Students who obtain negative outcomes must meet additional educational requirements within the first year. After the first enrolment period, universities may register various situations among students: standard enrolment, change of study course, abandonment of studies. Analysis of graduates of 2009/10 (approximately 420,500 units) regarding their academic career one year after enrolment in 2010/11 shows that $74 \%$ confirmed the enrolment in the same course, $14.8 \%$ of them changed course and $11.2 \%$ dropped out [2]. Statistics of Turin University in the three years before the start of Orient@mente services reflect the national situation: about $20 \%$ of students dropped out the studies during the first years, while the overall dropout rate is $13 \%$.
Successful transition from secondary school to University is a crucial phase for both students and institutions. In fact, a measure of the progress of university students must be weighted with the correctness of their study path choice. New technologies can be effective for university guidance, especially if they are supported by institutions [3]. Multimedia resources, digital interaction and online testing are available nowadays as a bridge connecting experts and learners. Development of MOOCs (Massive Open Online Courses) and the great variety of innovative tools integrated into LMS (Learning Management Systems) allow institutions to predict and find immediate solutions to new incoming needs [4].

Since 2014/15, University of Turin supports Orient@mente project within a strategic line on electronic services [5]. The main objective is to encourage students to consciously make their choice about academic studies. Orient@mente offers MOOCs through a public and open Moodle platform [6]. It includes information and guidance activities for University courses, free areas where students can prepare for admission tests, courses for revising basic knowledge acquired during high school, resources for foreign students and for students interested in the Erasmus program.

The outcomes of Orient@mente contributed in the choice of the University to provide autonomously the sessions of Test for the Assessment of Minimum Requirements (TARM) for scientific courses with open-entry starting from 2016/17 [7].

This paper discusses the methodologies adopted and the results obtained using the model in which the actions of Orient@mente are organized in synergy with the autonomous administration of TARM. 


\section{STATE OF THE ART}

One of the first challenges for academic institutions is to provide an appropriate process for admission to study courses. Before now, students facing the transition from secondary school to Turin University had to go through three steps:

- Orientation activities: traditional approach to students' orientation involves the organization of meetings by institutions. Disciplines are presented through talks and direct experiences usually condensed in half a day, involving large groups of students (considered that for many students their attention span could be short). Online pages of university courses contain institutional information but no guidance activities.

- Admission tests: for several years, each course of study decided criteria for admission tests independently. TARM examinations were distinct, tests involved different disciplines, test administration involved the use of web services or paper forms, some courses relied on outside agencies, other ones preferred to internally prepare the tests. This is a disadvantage for students: to maintain the option of enrolling in different courses, they need to prepare for distinct tests.

- First years exams: the lack of online courses for remedial purposes or to deepen one's knowledge is a disadvantage for students who enrol late and cannot attend university pre-courses.

Worldwide universities provide free and open access to educational content via MOOCs [8]: this is not effective in improving students' guidance if open learning plans are not designed for this objective but mainly for advertising purpose.

\section{THE MODEL}

University of Turin reinforces classic orientation activities for incoming students through a model involving interactive guidance to courses, testing with automatic evaluation and online tutoring. Actions of Orient@mente and the autonomous administration of TARM organized within the model have mutually reinforcing effects, and share tools and data with the pre-existing IT services of the University.

\section{A. Availability}

The model requires that Orient@mente and the autonomous TARM administration operate as continual services. Their actions exploit courses hosted on two distinct Moodle sites, both always accessible: the former is dedicated to students who intend to enrol at the University; the latter is open to everyone, in compliance with current laws [9].

\section{B. Integrated tools}

LMS admit the adoption of integrations. The University of Turin has great experience in the strategic use, development and maintenance of the following advanced tools integrated in Moodle for teaching and assessment.

1) General purpose Computer Algebra Systems

Advanced Computing Environment (ACE) are ideal tools not only for dealing with scientific disciplines, but also for every study involving a scientific approach. Explorative and interactive materials show students applications and tools that are similar to those from universities or the world of work.

Maple is a powerful and versatile ACE for numeric and symbolic computations, geometric visualizations in two and three dimensions and realization of interactive worksheets with embedded components. It is extensively used in Orient@mente, and in several university courses to study Mathematics. MapleNet is the online worksheet player: its integration turns worksheets into Moodle resources [10].

\section{2) Automatic Assessment Systems}

Systems for Automatic Assessment (AAS) help universities in monitoring students' results. Students can take great advantage in finding free and accessible tests validated by university experts. Reliability and versatility of the testing tool is fundamental for providing a lasting and evolving guidance.

Since Maple T.A. is based on the Maple engine, question creation benefits from a series of advanced features, such as numeric and symbolic computations, geometric visualizations in two and three dimensions, insertion of interactive components, algorithms and random generated variables. Maple T.A. integration allows assignments to be executed as Moodle resources, and students' results to be automatically updated in the gradebook [11].

\section{Organizational model}

Actions are based on team-working and modelled on the well-known Deming cycle [12]. They form a model generic enough to be adopted by any course of study (currently, 18 courses join TARM and Orient@mente). Fig. 1 shows the main participants and their roles.

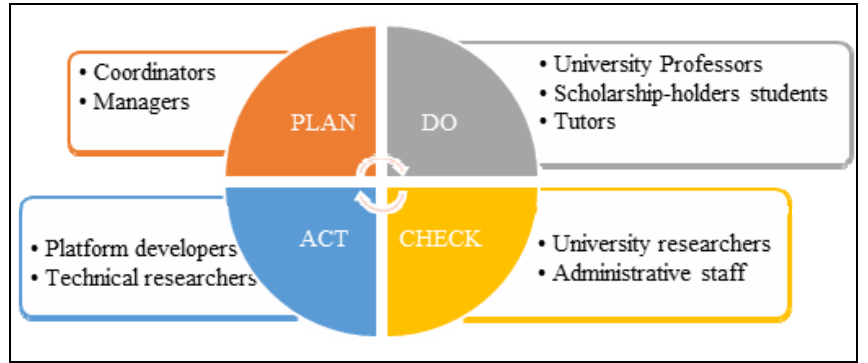

Fig. 1. Team-working synthesis for the organizational model.

- Managers together with coordinators perform studies to define suitable methodologies and instruments.

- Students with scholarships select and create learning materials, supported by professors and tutors who guarantee products' quality. Students from master degrees are selected, trained in the use of digital tools, coordinated by representatives of the university courses. While obtaining useful curricular experience, they contribute to the effectiveness of resources and tutoring thanks to their academic experience.

- Coordinators together with administrative staff oversee training, platform management, project communication, assistance and data management. 
- Platform developers and technical researchers cooperate to continuously adjust development of services according to the chosen methodologies, on the basis of user feedback and specific aspects.

\section{Action of Orientation to University}

Self-paced MOOCs can be designed to achieve the following objectives:

- supporting secondary school students in a more informed choice of the degree course, accompanying their choice since the fourth year of Secondary School;

- $\quad$ supporting those courses of study that have eliminated limited access;

- providing assistance in administering TARM to the degree courses with free access and admission tests to the degree courses with limited access.

1) MOOCs for Orientation

Orientation courses provide information about the courses of study and the careers that can be undertaken after graduation. Each MOOC contains:

- a course overview, approved by a commission of teachers belonging to each department, that provides essential information about the course attendance;

- interactive resources showing typical features and applications of the main subjects studied in the course, to help students understand if it meets their interests;

- resources and activities to orient students post university, including interviews with ex-students and main scenarios open to graduated students;

- open forums, where students can ask questions and obtain answers, meet other students in their similar situation and build a community for orientation;

- online tutoring, held through a web-conference tool according a set schedule: students can meet graduated or $\mathrm{PhD}$ students online and ask about their experience;

- $\quad$ sample tests to practice and get ready for the entry test.

2) MOOCs for Testing

Testing MOOCs are designed to verify students' basic knowledge and skills, and to enhance their awareness about their initial situation. Each MOOC covers one of the subjects involved in TARM or in admission tests, and it includes:

- a set of automatically graded tests;

- a preliminary video guide about how to perform a test and how to check feedback and results;

- $\quad$ survey about course appreciation and usefulness;

- $\quad$ personal gradebook: results and statistics from tests.

\section{E. Action of supports for University students}

Supporting MOOCs can be addressed to fulfill the following three aims:
- reducing the dropout rate of the first year of university;

- helping students pass their first university exams;

- $\quad$ spreading tertiary education.

Supporting MOOCs have a modular structure. Each course has an initial mind map which guides students through the course's content, with a focus on prerequisites. Course's content is organized into modules (topics) and sub-modules (arguments) with the following structure:

- lesson: theoretical explanations of the basis;

- explore: interactive activity to understand the lesson;

- applications of the topic to the real world or other disciplines, to see how theory turns into practice;

- exercises: a list of examples for autonomous execution;

- solutions of exercises to check execution's correctness;

- $\quad$ automatically graded assignment to interactively check the understanding of the lesson.

At the end of each module there is a summary test, automatically graded, to check the acquisition of knowledge and skills of the whole module.

Orient@mente hosts: four "Realignment courses", useful for strengthening proficiency and filling the gaps in the students' preparation in Biology, Chemistry, Physics and Mathematics; a MOOC - called "Mathematics in e-learning" that gives the opportunity to study fundamental concepts of basic Math interactively, and which is suitable as a first-year elearning course on basic mathematics (different degree programs can choose which parts must be mastered by students); MOOCs for learning Italian dedicated to foreign students; a MOOC orienting towards Erasmus experience.

\section{F. Autonomous TARM administration}

For degree courses with TARM, students can enrol only after taking the test. Failing TARM (that is, taking it with no success) has repercussions on the student's university career. Each course of study fixes autonomously thresholds that students need to meet. Students who do not pass TARM and/or who need to complete some additional activities (Supplementary Educational Obligations assigned by their course of study) have certain possibilities suspended, such as the compilation of the career plan or the reservation and taking of exams.

TARM involves the use of IT services computer labs where students take the tests. University teaching and technical staff supervise lab sessions to guarantee the correct execution of tests. TARM is divided into three typologies of session:

- $\quad$ anticipated session - usually in spring - dedicated to students of the fifth year of secondary school;

- ordinary session held during the enrolment period and dedicated to students enrolling to University;

- remedial session dedicated to students who are already enrolled at the University. 
TARM Moodle platform - based on the tools of Orient@mente - is conceived to assist beginner students in managing their career through a personal page. For this purpose, new integrations were built to connect Moodle to the university system. Each student $\log$ on to Moodle with his university portal credentials, and is directed to a personal page to manage the TARM process through three main blocks:

- booking an examination session: a summary of personal booking reservations (active or expired) with location and date of the test is displayed. A PDF file with all the previous indications is available for download, and may be required on the day of the test for the recognition of the candidate together with a photo ID. It embeds a cryptographic, tamper-evident QR-code that shows and validates the active booking;

- access the test: a link to take the TARM. A unique enrolment password to access the test is required. This is communicated during the lab sessions;

- results summary: TARM sessions scores are displayed. The student can download a PDF file with the scores that contains a cryptographic encoded, tamper-evident $\mathrm{QR}$ code that shows and validates the scores.

During TARM registration, students are asked to indicate preferences for study courses for eventual future enrolment.

The structure of the TARM is the same for all the courses of study involved. It is composed of five distinct tests with multiple-choice questions, as summarized in TABLE I.

TABLE I. DISCIPLINES OF TARM

\begin{tabular}{|l|l|l|}
\hline \multicolumn{1}{|c|}{ Discipline } & Questions & Available time \\
\hline Biology & 10 & 20 minutes \\
\hline Chemistry & 10 & 20 minutes \\
\hline Physics & 10 & 20 minutes \\
\hline Comprehension of Scientific Texts & 5 & 15 minutes \\
\hline Basic Mathematics & 25 & 75 minutes \\
\hline
\end{tabular}

Each student chooses how to perform the TARM at his own time pace: he chooses which, how many and the order of the tests to take, without having to wait. Each correct answer adds one point to the total score of the test. There are no penalties for wrong answers. Each course of study sets a threshold for the test of each discipline. Students compare their results with the thresholds of their university courses.

The establishment of a unique examination jointly with different thresholds allow students to deal with the prerequisites required for a degree program, which is a fundamental action for orientation. Professors from different courses of study contributed to the realization of shared collections of multiple-choice questions, written in the AAS with a text editor in the LaTeX language.

\section{G. Support and communication}

The main information is displayed on the official university web pages. Communications to students are managed consistently by the same support group which operates for both TARM and Orient@mente. Piedmont and Aosta Valley Regional Education Communication Offices helped in communicating to schools. Notes from general managers of the USR Piemonte were used for advertising the Orient@mente platform to the first beneficiaries [13].

Within Moodle, students can consult relevant information about TARM exams. Delivering communications mainly through a personal page prevents potential problems that can occur if information systems relied solely on e-mail dispatch.

\section{Properties}

The model is characterized by the following properties.

- Accessibility: platforms adopt the high-legibility font "EasyReading" designed for people with dyslexia [14]. People with disabilities or specific learning disorders can benefit from customized support tools during tests.

- Adaptability: the structure and tools chosen for the model make it applicable to different university requirements and students' learning approaches.

- Consistency: since the first approach to the university, students become familiar with systems that will accompany them throughout their careers.

- Control: institutions can perform ongoing analysis and continually adapt actions to students' needs; students are facilitated in autonomously overcoming their insecurities by immediate feedback.

- Convenience: the integration of multiple resources adapts to the development of and ongoing research on new technology.

- Free availability: learners from all over the world can benefit from materials distributed on public pages.

- Efficiency: guidance starts from the first contacts between learners and institutions.

- High-quality: every point of contact between students and university is managed by highly-qualified personnel continuously interacting.

- Sustainability: integrated tools and the collaboration between experts from distinct ranges of expertize guarantee contained costs for production of material and services, as well as the costs for students.

- Usefulness: secondary school students are provided with advanced tools to make informed choices at the time of enrolment; this preventative action positively affects institutions, improving the quality of courses offered.

\section{RESULTS AND DISCUSSION}

Students' experience is broad and involves several distinct aspects: the joint participation of different institutional entities is a key point to reach an operative data analysis. Integrations with LMS can encourage the collection and comparison of students' results and academic career. 


\section{A. Subscriptions, logins and tests in Orient@mente}

Analysis of Orient@mente platform usage helps estimate the effectiveness of the guidance to the university. In proximity of 2016 TARM sessions, Orient@mente platform registered peaks of the number of subscribed users and unique logins per month, as shown by Fig. 2 .

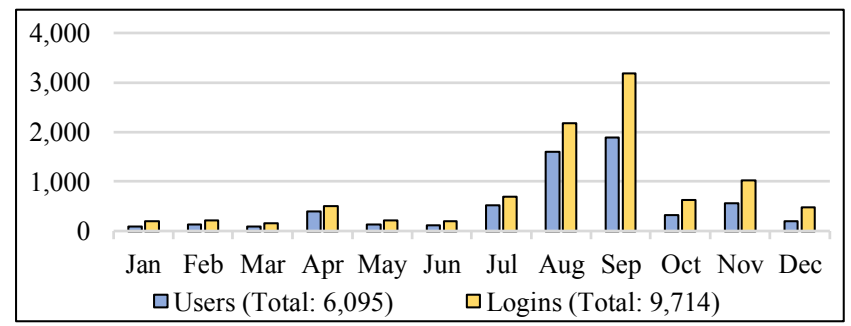

Fig. 2. Users subscriptions and unique logins for Orient@mente in 2016.

A similar trend holds for the number of tests for exam preparation performed in 2016, as shown by Fig. 3 .

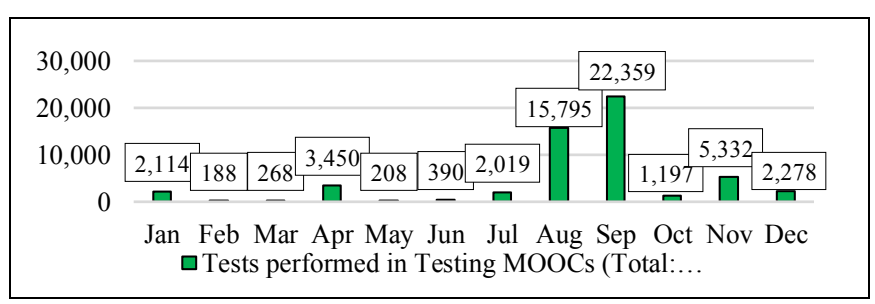

Fig. 3. Tests performed per month in platform Orient@mente in 2016.

TABLE II. shows the number of users who subscribed to Orient@mente MOOCs, the related number of available tests, and the amount of attempts made to courses' tests.

TABLE II._USERS, TESTS, ATTEMPTS FOR MOOCS OF ORIENT@MENTE

\begin{tabular}{|l|l|l|l|}
\hline \multicolumn{1}{|c|}{ MOOC } & Users & Tests & \multicolumn{1}{c|}{ Attempts } \\
\hline Biology & 2249 & 10 & 12327 \\
\hline Biology (Realignment course) & 434 & 14 & 892 \\
\hline Basic Mathematics & 2809 & 12 & 12075 \\
\hline Chemistry & 1941 & 10 & 10283 \\
\hline Chemistry (Realignment course) & 397 & 9 & 427 \\
\hline Comprehension of Scientific Texts & 1711 & 8 & 5784 \\
\hline Current affairs, General knowledge & 1709 & 3 & 2822 \\
\hline Earth Science & 330 & 3 & 572 \\
\hline Logic & 1220 & 4 & 2435 \\
\hline Mathematics and Problems & 762 & 1 & 261 \\
\hline Mathematics (Realignment course) & 768 & 27 & 1456 \\
\hline Physics & 1954 & 10 & 9039 \\
\hline Physics (Realignment course) & 535 & 25 & 1783 \\
\hline
\end{tabular}

\section{B. Anonymous questionnaires for evaluation}

Participants and students' feedback - as well as the stability of the platform and the tools adopted - show the effectiveness of the model. Data about the level of appreciation of TARM and Orient@mente are collected by anonymous questionnaires.
1) Questionnaire for the evaluation of Orient@mente

Fig. 4 shows a high approval of Orient@mente services: the helpfulness in preparing for the admission tests, the usefulness and the ease of use were evaluated by at least 3 points out of 5 by more than $75 \%$ of the interviewed.

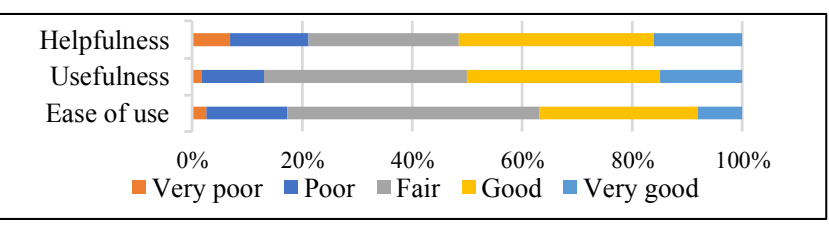

Fig. 4. Results from the Questionnaire for evaluation of Orient@mente

2) Questionnaire for the evaluation of 2016 TARM

High appreciation of 2016 TARM by the university personnel involved is resumed in TABLE III.

TABLE III. QUESTIONNAIRE ABOUT 2016 TARM

\begin{tabular}{|l|l|}
\hline \multicolumn{1}{|c|}{$\begin{array}{c}\text { Question } \\
(\mathbf{1}=\text { Poor, 2 = Fair, 3 = Average, } \mathbf{4}=\text { Good, 5 = Excellent })\end{array}$} & $\begin{array}{c}\text { Average } \\
\text { rate }\end{array}$ \\
\hline Completeness of information provided to workers & 4.6 \\
\hline Completeness of the information to students in the classroom & 4.8 \\
\hline Competence and courtesy of operators & 5 \\
\hline Availability and support of the operator & 4.7 \\
\hline Management of students with learning difficulties or disability & 4.8 \\
\hline Administration and timing of the examinations & 4.7 \\
\hline Regularity in tests' performances & 4.7 \\
\hline Management of any problems arising during the turn & 4.8 \\
\hline Times of turn conduct & 4.7 \\
\hline Delivery method of testing TARM & 4.6 \\
\hline Stability of TARM platform & 4.5 \\
\hline Usability of the automatic evaluation system for students & 4.4 \\
\hline Total effectiveness & 4.6 \\
\hline
\end{tabular}

The TARM questionnaire also inquired "positive elements encountered during 2016/2017 TARM sessions". Among the free answers are: "effectiveness of online mode"; "readiness in notifying results"; "user-friendly web interface"; "timeliness of students' support"; "competence and preparation of personnel who handled the platform and of the technical staff in labs".

3) Direct feedback from testing courses of Orient@mente

At the end of each course test there is a survey to gather suggestions and a free feedback of the level of appreciation of Orient@mente: currently, 96\% of the submitters answered "Yes" to the question "Do you consider Orient@mente a useful service offered by University of Turin?".

\section{Students at University of Turin in 2015/16 and 2016/17}

Measures on the users of Orient@mente, compared to the enrolled students, were helpful in promoting changes to special or negative cases after the first enrolment period.

When combining the registration mail fields from the Orient@mente users' list and the list of students who enrolled to University of Turin, 872 matches occur. TABLE IV. shows 
the number of students who performed at least one test on Orient@mente testing courses in the periods within TARM sessions. Success rate is equal or above $74 \%$ for every session.

TABLE IV. SUCCESS IN PASSING TARM SESSIONS

\begin{tabular}{|l|l|l|}
\hline Tarm session & Matches $^{\mathrm{a}}$ & Matches $^{\mathrm{a}}$ who passed TARM session \\
\hline 18-22 Apr & 76 & 62 \\
\hline 29 Aug - 4 Oct & 658 & 505 \\
\hline 7-11 Nov & 91 & 70 \\
\hline 12-16 Dec & 116 & 86 \\
\hline Total & 941 & 723 \\
\hline
\end{tabular}

a. Students whose mail of university portal credentials is in Orient@mente users' mail list.

Restricting the search to the number of students who passed the TARM at the first attempt, the overall number among all the sessions is 669 students out of 872 , that is $77 \%$.

Data collected from TARM booking can improve the accuracy of estimations of how many students will enrol to the university courses involved. During the anticipated session, there was a first mandatory choice, and two other optional choices (without order of importance). Considering the students who effectively enrolled to the University of Turin, $93 \%$ of them chose to enrol to one of the three choices indicated. Considering only the students that have indicated only one preference, $95 \%$ of them chose to enrol to the preferred course of study. After the ordinary session, considering the students who effectively enrolled to the University of Turin, $90 \%$ of them chose to enrol to the firstchoice of course of study. About half of the remaining enrolled to the second choice.

\section{CONCLUSIONS}

A reorganization of the evaluation modality that takes into account specific needs, supported by targeted orientation actions, should prevent dropout in the study program. Nevertheless, facilitating the maturation of the students' sense of responsibility towards their academic career is a concurrent consequence of the improvements in the organization of university's actions.

The model is applicable and sharable among different universities, and could be spread by regional directorate and MIUR: services are connected, while the autonomy of courses of study is maintained within the collaborative workflow regardless of differences among the disciplines involved.

After enrolment, realignment courses can be used by students during the remedial TARM phase both as a self-study resource and as a resource indicated by the course of study. In fact, the latter assigns additional tasks to be performed via the MOOCs within the supplementary educational obligation.

The AAS contains tools for monitoring success rate of questions and other statistics on the relevance of questions within the test. An a-posteriori analysis of the questions databases and of all the results could ensure that the distribution of the questions over the tests was fair as regards the difficulty level and the topics covered.

For future development and research, a new structure of admission tests could also be taken into account. For instance, questions of different typology from multiple choice can be used for students' assessment via the TARM. This would be possible thanks to the on-going experience in the creation of questions via the AAS for Orient@mente's MOOCs.

\section{ACKNOWLEDGMENT}

The authors thank Rector Prof. G. Ajiani and Pro-Rector Prof. E. Barberis of University of Turin, who enthusiastically supported the project, Dr. M. Bruno and the staff of Board of Education and Student Services, Ing. A. Saccà and the staff of Portal Management, ICT services of Department of Computer Science, teachers and technical-administrative personnel at Turin University who collaborated at different levels.

\section{REFERENCES}

[1] MIUR, Decreto n. 270 del 22/10/2004, Modifiche al regolamento recante norme concernenti l'autonomia didattica degli atenei, approvato il 3/11/1999, n. 509, 2004.

[2] MIUR, Focus “Gli immatricolati nell'a.a. 2014/2015", 2015.

[3] SMEs \& e-LEARNING Project, European-wide e-Learning Recognition Review Report, 2015.

[4] R. Pyke, Addressing first year university Mathematics and the transition from high school at Simon Fraser University. 12th International Congress on Mathematical Education. Seoul, Korea, 2012.

[5] A. Barana, A. Bogino, M. Fioravera, M. Marchisio, S. Rabellino, Open platform of self-paced MOOCs for the continual improvement of academic guidance and knowledge strengthening in tertiary education, Atti di EMEMITALIA, 2016.

[6] A. Barana, A. Bogino, M. Fioravera, M. Marchisio, S. Rabellino, "Digital Support for University Guidance and Improvement of Study Results", Procedia-Social and Behavioral Sciences, 228, 547-552, 2016.

[7] A. Barana, A. Bogino, M. Fioravera, M. Marchisio, S. Rabellino, La piattaforma Moodle al Servizio del Test di Accertamento dei Requisiti Minimi per l'Ingresso in Università, Atti di EMEMITALIA, 2016.

[8] B. Grainger, Introduction to MOOCs: avalanche, illusion or augmentation? Policy brief of unesco institute for information technologies in education, 2013.

[9] D. L.196, Codice in materia di protezione dei dati personali, 30/06/2003.

[10] M. Baldoni, A. Cordero, S. Coriasco, M. Marchisio, Studiare la Matematica con Moodle, Maple, MapleNet e MapleTA: dalla lezione alla valutazione, E-Learning con Moodle in Italia: una sfida tra presente, passato e futuro, Seneca Edizioni, 2011, 299-316

[11] A. Barana, M. Marchisio, S. Rabellino, Automated Assessment in Mathematics, COMPSAC Symposium on Computer Education and Learning Technologies, Taichung, 2015.

[12] T. Walasek, Z. Kucharczyk, D. Morawska-Walasek, Assuring quality of an e-learning project through the PDCA approach. Archives of Materials Science and Engineering 48/1, 56-61, 2011.

[13] USR Piemonte, Progetto Orient@mente: strumento di orientamento al mondo universitario dell'Università di Torino, Nota n. 4223, 8/06/2015.

[14] EasyReading. From www.easyreading.it. 